CERN-TH/2001-222

hep-ph/0111164

\title{
Direct Higgs production and jet veto at the Tevatron and the LHC in NNLO QCD"
}

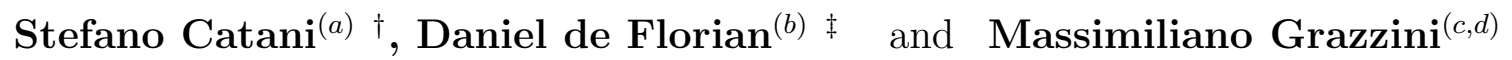 \\ ${ }^{(a)}$ Theory Division, CERN, CH-1211 Geneva 23, Switzerland \\ (b) Departamento de Física, FCEYN, Universidad de Buenos Aires, (1428) Pabellón 1 Ciudad \\ Universitaria, Capital Federal, Argentina \\ (c) Dipartimento di Fisica, Università di Firenze, I-50019 Sesto Fiorentino, Florence, Italy \\ ${ }^{(d)}$ INFN, Sezione di Firenze, I-50019 Sesto Fiorentino, Florence, Italy
}

\begin{abstract}
We consider Higgs boson production through gluon-gluon fusion in hadron collisions, when a veto is applied on the transverse momenta of the accompanying hard jets. We compute the QCD radiative corrections to this process at NLO and NNLO. The NLO calculation is complete. The NNLO calculation uses the recently evaluated NNLO soft and virtual QCD contributions to the inclusive cross section. We find that the jet veto reduces the impact of the NLO and NNLO contributions, the reduction being more sizeable at the LHC than at the Tevatron.
\end{abstract}

CERN-TH/2001-222

November 2001

\footnotetext{
*This work was supported in part by the EU Fourth Framework Programme "Training and Mobility of Researchers", Network "Quantum Chromodynamics and the Deep Structure of Elementary Particles", contract FMRX-CT98-0194 (DG 12 - MIHT).

${ }^{\dagger}$ On leave of absence from INFN, Sezione di Firenze, Florence, Italy.

${ }^{\ddagger}$ Partially supported by CONICET and Fundación Antorchas
} 


\section{Introduction}

The mechanism of electroweak symmetry breaking is one of the main issues in high-energy particle physics. In the Standard Model (SM) and in its supersymmetric extensions the mechanism is accomplished by elementary scalar doublets. The Higgs boson(s) [1] are thus a fundamental ingredient of the theory, and their search is the main goal of current and future colliders.

The direct search at LEP implies a lower limit of $M_{H}>114.1 \mathrm{GeV}$ (at 95\% CL) [2] on the mass $M_{H}$ of the SM Higgs boson, and shows an excess of events, which may indicate the production of a Higgs boson with mass near $115 \mathrm{GeV}$ [3, 4, 2]. Global SM fits to electroweak precision measurements favour a light Higgs $\left(M_{H} \lesssim 200 \mathrm{GeV}\right)$ [5].

After the end of the LEP era, the search for the Higgs boson will be carried out at hadron colliders. Depending on the luminosity delivered to the CDF and D0 detectors during the forthcoming Run II, the Tevatron experiments can yield evidence for a Higgs boson with $M_{H} \lesssim 180 \mathrm{GeV}$ and may be able to discover (at the $5 \sigma$ level) a Higgs boson with $M_{H} \lesssim 130 \mathrm{GeV}$ [6]. At the LHC, the SM Higgs boson can be discovered over the full mass range up to $M_{H} \sim 1 \mathrm{TeV}$ after a few years of running [7].

The dominant mechanism for SM Higgs boson production at hadron colliders is gluon-gluon fusion through a heavy-quark (top-quark) loop [8]. This mechanism is often called direct Higgs production, to distinguish it from associated production of Higgs boson and vector bosons, heavy quarks, jets, and so forth.

At the LHC [9], gg fusion exceeds all the other production channels by a factor decreasing from 8 to 5 when $M_{H}$ increases from 100 to $200 \mathrm{GeV}$. When $M_{H}$ approaches $1 \mathrm{TeV}, g g$ fusion still provides about $50 \%$ of the total production cross section. At low values of $M_{H}$ the dominant decay mode is $H \rightarrow b \bar{b}$, but it is swamped by the QCD background. This decay mode is thus exploited [7] in the low-mass range $100 \mathrm{GeV} \lesssim M_{H} \lesssim 120 \mathrm{GeV}$ only in the case of the associated production $H t \bar{t}$. In the case of direct production, the most important decay channels for Higgs searches [7] are the rare decay $H \rightarrow \gamma \gamma$ in the low-mass range $100 \mathrm{GeV} \lesssim M_{H} \lesssim 140 \mathrm{GeV}$, and the channel $H \rightarrow Z Z \rightarrow 4 l$ in the mass range $130 \mathrm{GeV} \lesssim M_{H} \lesssim 700 \mathrm{GeV}$. In the intermediate-mass region $150 \mathrm{GeV} \lesssim M_{H} \lesssim 190 \mathrm{GeV}$, direct Higgs production followed by the decay $H \rightarrow W W \rightarrow l^{+} l^{-} \nu \bar{\nu}$ is also relevant. The strong angular correlations of the final-state leptons from $W$ decay are an important ingredient for this discovery channel [10].

At the Tevatron, $g g$ fusion remains the main production channel [6], but it is experimentally less important than at the LHC because the decay rate $H \rightarrow \gamma \gamma$ is too low to be observed. When $M_{H} \lesssim 135 \mathrm{GeV}$, the most promising discovery mechanism [6] is thus the associated production $\left(q \bar{q} \rightarrow V^{*} \rightarrow H V\right)$ of the Higgs boson with a vector boson $V(V=W$ or $Z)$, whose leptonic decay provides the trigger for the signal from $H \rightarrow b \bar{b}$. Nevertheless, since the decays $H \rightarrow$ $W^{*} W^{*}, Z^{*} Z^{*}$ have increasing branching fractions for $M_{H} \gtrsim 130 \mathrm{GeV}, g g \rightarrow H \rightarrow W^{*} W^{*}, Z^{*} Z^{*}$ are the natural channels to consider when $140 \mathrm{GeV} \lesssim M_{H} \lesssim 180 \mathrm{GeV}$. In particular, the decay mode $W^{*} W^{*} \rightarrow l^{+} l^{-} \nu \bar{\nu}$ is quite important [6], since it is cleaner than $W^{*} W^{*} \rightarrow l \nu j j$, and the decay rate $H \rightarrow W^{*} W^{*}$ is higher than $H \rightarrow Z^{*} Z^{*}$ by about one order of magnitude.

An important background for the direct Higgs signal $H \rightarrow W^{*} W^{*} \rightarrow l^{+} l^{-} \nu \bar{\nu}$ is $t \bar{t}$ production $\left(t W\right.$ production is also important at the LHC), where $t \rightarrow l \bar{\nu} b$, thus leading to $b$ jets with high $p_{T}$ 
in the final state. If the $b$ quarks are not identified, a veto cut on the transverse momenta of the jets accompanying the final-state leptons can be applied to enhance the signal/background ratio. Imposing a jet veto turns out to be essential, both at the Tevatron [6, 11] and at the LHC [7], to cut the hard $b$ jets arising from this background process.

The next-to-leading order (NLO) QCD corrections to $g g$ fusion are large [12, 13, 14]. Approximate evaluations [15] of higher-order terms suggest that their effect can still be sizeable. The computation of the next-to-next-to-leading order (NNLO) corrections is thus important to better estimate the cross section for direct Higgs production.

Recently two groups [16, 17] have performed a first step in this direction, by evaluating the soft and virtual contributions to the NNLO partonic cross section $\hat{\sigma}(g g \rightarrow H+X)$ in the large$M_{\text {top }}$ approximation. Our calculation [16] was performed by combining Harlander's result [18] for the two-loop amplitude $g g \rightarrow H$ with the soft factorization formulae for tree-level [19, 20] $(g g \rightarrow H g g, H q \bar{q})$ and one-loop [21, 22] $(g g \rightarrow H g)$ amplitudes, and then using the technique of Ref. [23]. The independent calculation of Ref. [17] used a different method, and the analytical results fully agree.

In Ref. [16] we also studied the quantitative impact of the soft and virtual NNLO contributions on direct Higgs boson production at the LHC. This was consistently done by using the recent MRST2000 set of parton distribution functions [24], which includes (approximated [25]) NNLO densities. In this paper we first perform an analogous studyl at the Tevatron Run II, and we show that the soft and virtual contributions are expected to give a fairly good estimate of the complete NNLO result.

In the second part of the paper we study the effect of a jet veto on direct Higgs production beyond the leading order (LO). We present new NLO and NNLO calculations for the vetoed cross section both at the Tevatron and at the LHC. These calculations are performed by subtracting the LO (NLO) cross section for the production of Higgs plus jets from the corresponding inclusive NLO (NNLO) cross section. The LO and NLO subtracted cross section for the production of Higgs plus jets is evaluated in the large- $M_{\text {top }}$ limit, but without any soft and virtual approximations. At LO we derive analytical expressions, while at the NLO we use the numerical program of Ref. [27].

The paper is organized as follows. In Sect. 2 we recall the theoretical framework and the analytical results for the soft and virtual NNLO corrections. In Sect. 3 we present the results for inclusive Higgs production at the Tevatron Run II. In Sect. 4 we present the results obtained by applying a jet veto on the inclusive cross section, both at the Tevatron Run II and at the LHC. Our conclusions are summarized in Sect. 5 .

\footnotetext{
${ }^{\dagger}$ Part of these results was anticipated in Ref. [26].
} 


\section{Inclusive QCD cross section at NNLO}

We consider the collision of two hadrons $h_{1}$ and $h_{2}$ with centre-of-mass energy $\sqrt{s}$. The inclusive cross section for the production of the SM Higgs boson can be written as

$$
\begin{aligned}
\sigma\left(s, M_{H}^{2}\right)= & \sum_{a, b} \int_{0}^{1} d x_{1} d x_{2} f_{a / h_{1}}\left(x_{1}, \mu_{F}^{2}\right) f_{b / h_{2}}\left(x_{2}, \mu_{F}^{2}\right) \int_{0}^{1} d z \delta\left(z-\frac{\tau_{H}}{x_{1} x_{2}}\right) \\
& \cdot \sigma_{0} z G_{a b}\left(z ; \alpha_{\mathrm{S}}\left(\mu_{R}^{2}\right), M_{H}^{2} / \mu_{R}^{2} ; M_{H}^{2} / \mu_{F}^{2}\right),
\end{aligned}
$$

where $\tau_{H}=M_{H}^{2} / s$, and $\mu_{F}$ and $\mu_{R}$ are factorization and renormalization scales, respectively. The parton densities of the colliding hadrons are denoted by $f_{a / h}\left(x, \mu_{F}^{2}\right)$ and the subscript $a$ labels the type of massless partons $\left(a=g, q_{f}, \bar{q}_{f}\right.$, with $N_{f}$ different flavours of light quarks). We use parton densities as defined in the $\overline{\mathrm{MS}}$ factorization scheme.

From Eq. (11) the cross section $\hat{\sigma}_{a b}$ for the partonic subprocess $a b \rightarrow H+X$ at the centre-of-mass energy $\hat{s}=x_{1} x_{2} s=M_{H}^{2} / z$ is

$$
\hat{\sigma}_{a b}\left(\hat{s}, M_{H}^{2}\right)=\frac{1}{\hat{s}} \sigma_{0} M_{H}^{2} G_{a b}(z)=\sigma_{0} z G_{a b}(z),
$$

where the term $1 / \hat{s}$ corresponds to the flux factor and leads to an overall $z$ factor. The Born-level cross section $\sigma_{0}$ and the hard coefficient function $G_{a b}$ arise from the phase-space integral of the matrix elements squared.

Equation (四) can also be recast in the form

$$
\sigma\left(s, M_{H}^{2}\right)=\sigma_{0} \tau_{H} \sum_{a, b} \int_{\tau_{H}}^{1} \frac{d \tau}{\tau} \mathcal{L}_{a b / h_{1} h_{2}}\left(\tau, \mu_{F}^{2}\right) G_{a b}\left(\tau_{H} / \tau\right),
$$

where the function

$$
\mathcal{L}_{a b / h_{1} h_{2}}\left(\tau, \mu_{F}^{2}\right)=\int_{\tau}^{1} \frac{d x}{x} f_{a / h_{1}}\left(x, \mu_{F}^{2}\right) f_{b / h_{2}}\left(\tau / x, \mu_{F}^{2}\right)
$$

is the parton luminosity that weights the event initiated by partons $a$ and $b$.

The incoming partons $a, b$ couple to the Higgs boson through heavy-quark loops and, therefore, $\sigma_{0}$ and $G_{a b}$ also depend on the masses $M_{Q}$ of the heavy quarks. The Born-level contribution $\sigma_{0}$ is 8

$$
\sigma_{0}=\frac{G_{F}}{288 \pi \sqrt{2}}\left|\sum_{Q} A_{Q}\left(\frac{4 M_{Q}^{2}}{M_{H}^{2}}\right)\right|^{2},
$$

where $G_{F}=1.16639 \times 10^{-5} \mathrm{GeV}^{-2}$ is the Fermi constant, and the amplitude $A_{Q}$ is given by

$$
\begin{aligned}
A_{Q}(x) & =\frac{3}{2} x[1+(1-x) f(x)], \\
f(x) & = \begin{cases}\arcsin ^{2} \frac{1}{\sqrt{x}}, & x \geq 1 \\
-\frac{1}{4}\left[\ln \frac{1+\sqrt{1-x}}{1-\sqrt{1-x}}-i \pi\right]^{2}, & x<1\end{cases}
\end{aligned}
$$


In the following we limit ourselves to considering the case of a single heavy quark, the top quark, and $N_{f}=5$ light-quark flavours. We always use $M_{\text {top }}\left(M_{\text {top }}=176 \mathrm{GeV}\right)$ to denote the on-shell pole mass of the top quark.

The coefficient function $G_{a b}$ in Eq. (1) is computable in QCD perturbation theory according to the expansion

$$
\begin{aligned}
& G_{a b}\left(z ; \alpha_{\mathrm{S}}\left(\mu_{R}^{2}\right), M_{H}^{2} / \mu_{R}^{2} ; M_{H}^{2} / \mu_{F}^{2}\right)=\alpha_{\mathrm{S}}^{2}\left(\mu_{R}^{2}\right) \sum_{n=0}^{+\infty}\left(\frac{\alpha_{\mathrm{S}}\left(\mu_{R}^{2}\right)}{\pi}\right)^{n} G_{a b}^{(n)}\left(z ; M_{H}^{2} / \mu_{R}^{2} ; M_{H}^{2} / \mu_{F}^{2}\right) \\
& =\alpha_{\mathrm{S}}^{2}\left(\mu_{R}^{2}\right) G_{a b}^{(0)}(z)+\frac{\alpha_{\mathrm{S}}^{3}\left(\mu_{R}^{2}\right)}{\pi} G_{a b}^{(1)}\left(z ; \frac{M_{H}^{2}}{\mu_{R}^{2}} ; \frac{M_{H}^{2}}{\mu_{F}^{2}}\right)+\frac{\alpha_{\mathrm{S}}^{4}\left(\mu_{R}^{2}\right)}{\pi^{2}} G_{a b}^{(2)}\left(z ; \frac{M_{H}^{2}}{\mu_{R}^{2}} ; \frac{M_{H}^{2}}{\mu_{F}^{2}}\right)+\mathcal{O}\left(\alpha_{\mathrm{S}}^{5}\right),
\end{aligned}
$$

where the (scale-independent) LO contribution is

$$
G_{a b}^{(0)}(z)=\delta_{a g} \delta_{b g} \delta(1-z) .
$$

Throughout the paper we work in the framework of the large- $M_{\text {top }}$ expansion, where one can exploit the effective-lagrangian approach [28, 29, 15] to embody the heavy-quark loop in an effective vertex. However, unless otherwise stated, we include in $\sigma_{0}$ the full dependence on $M_{\text {top }}$. This approximation [14, 15] turns out to be very good when $M_{H} \leq 2 M_{\text {top }}$, and it is still accuratef to better than $10 \%$ when $M_{H} \lesssim 1 \mathrm{TeV}$.

The NLO coefficients $G_{a b}^{(1)}(z)$ are known [12, 14]. Their explicit expressions (see e.g. Ref. [16]) contain three kinds of contributions:

- Virtual and soft contributions, which are respectively proportional to $\delta(1-z)$ and to the distributions $\mathcal{D}_{i}(z)$, where

$$
\mathcal{D}_{i} \equiv\left[\frac{\ln ^{i}(1-z)}{1-z}\right]_{+} .
$$

These are the most singular terms when $z \rightarrow 1$.

- Purely collinear logarithmic contributions of the type $\ln ^{i}(1-z)$. These contributions give the next-to-dominant singular terms when $z \rightarrow 1$.

- Hard contributions, which are finite in the limit $z \rightarrow 1$.

The soft-virtual (SV) approximation is defined [16] by keeping only the terms proportional to $\delta(1-z)$ and $\mathcal{D}_{i}(z)$ in the coefficient $G_{a b}$. In this approximation only the $g g$ channel contributes and we have

$$
\begin{aligned}
G_{a b}^{(1) \mathrm{SV}}\left(z ; M_{H}^{2} / \mu_{R}^{2} ; M_{H}^{2} / \mu_{F}^{2}\right) & =\delta_{a g} \delta_{b g}\left[\delta(1-z)\left(\frac{11}{2}+6 \zeta(2)+\frac{33-2 N_{f}}{6} \ln \frac{\mu_{R}^{2}}{\mu_{F}^{2}}\right)\right. \\
& \left.+6 \mathcal{D}_{0} \ln \frac{M_{H}^{2}}{\mu_{F}^{2}}+12 \mathcal{D}_{1}\right] .
\end{aligned}
$$

†The accuracy of this approximation when $M_{H} \lesssim 2 M_{\text {top }}$ may not be accidental. In fact, as discussed in the following, the main part of the QCD corrections to direct Higgs production is due to parton radiation at relatively low transverse momenta. Such radiation is weakly sensitive to the mass of the heavy quark in the loop. 
The NNLO coefficients $G_{a b}^{(2)}$ are not yet completely known, but their SV approximation has been computed in Refs. [16, 17]. It reads:

$$
\begin{aligned}
G_{g g}^{(2) S \mathrm{~V}}\left(z ; M_{H}^{2} / \mu_{R}^{2}, M_{H}^{2} / \mu_{F}^{2}\right) & =\delta(1-z)\left[\frac{11399}{144}+\frac{133}{2} \zeta(2)-\frac{9}{20} \zeta(2)^{2}-\frac{165}{4} \zeta(3)\right. \\
& +\left(\frac{19}{8}+\frac{2}{3} N_{f}\right) \ln \frac{M_{H}^{2}}{M_{\mathrm{top}}^{2}}+N_{f}\left(-\frac{1189}{144}-\frac{5}{3} \zeta(2)+\frac{5}{6} \zeta(3)\right) \\
& +\frac{\left(33-2 N_{f}\right)^{2}}{48} \ln ^{2} \frac{\mu_{F}^{2}}{\mu_{R}^{2}}-18 \zeta(2) \ln ^{2} \frac{M_{H}^{2}}{\mu_{F}^{2}} \\
& +\left(\frac{169}{4}+\frac{171}{2} \zeta(3)-\frac{19}{6} N_{f}+\left(33-2 N_{f}\right) \zeta(2)\right) \ln \frac{M_{H}^{2}}{\mu_{F}^{2}} \\
& \left.+\left(-\frac{465}{8}+\frac{13}{3} N_{f}-\frac{3}{2}\left(33-2 N_{f}\right) \zeta(2)\right) \ln \frac{M_{H}^{2}}{\mu_{R}^{2}}\right] \\
+ & \mathcal{D}_{0}\left[-\frac{101}{3}+33 \zeta(2)+\frac{351}{2} \zeta(3)+N_{f}\left(\frac{14}{9}-2 \zeta(2)\right)+\left(\frac{165}{4}-\frac{5}{2} N_{f}\right) \ln \frac{M_{H}^{2}}{\mu_{F}^{2}}\right. \\
& \left.-\frac{3}{2}\left(33-2 N_{f}\right) \ln \frac{M_{H}^{2}}{\mu_{F}^{2}} \ln \frac{M_{H}^{2}}{\mu_{R}^{2}}+\left(\frac{133}{2}-45 \zeta(2)-\frac{5}{3} N_{f}\right) \ln \frac{M_{H}^{2}}{\mu_{F}^{2}}\right] \\
+ & \mathcal{D}_{1}\left[133-90 \zeta(2)-\frac{10}{3} N_{f}+36 \ln ^{2} \frac{M_{H}^{2}}{\mu_{F}^{2}}+\left(33-2 N_{f}\right)\left(2 \ln \frac{M_{H}^{2}}{\mu_{F}^{2}}-3 \ln \frac{M_{H}^{2}}{\mu_{R}^{2}}\right)\right] \\
+ & \mathcal{D}_{2}\left[-33+2 N_{f}+108 \ln \frac{M_{H}^{2}}{\mu_{F}^{2}}\right] \\
+ & -32
\end{aligned}
$$

To give a rough idea of the numerical hierarchy of the LO, NLO and NNLO contributions, we can set $\mu_{F}=\mu_{R}=M_{H}=115 \mathrm{GeV}$ and $\alpha_{\mathrm{S}}\left(M_{H}\right)=0.112$. The first three terms in the expansion (17) thus give

$$
\begin{aligned}
G_{g g}^{\mathrm{SV}}(z)=\alpha_{\mathrm{S}}^{2}\{\delta(1 & -z)(1+0.548+0.105) \\
& +\mathcal{D}_{0}(0+0.283) \\
& +\mathcal{D}_{1}(0+0.428-0.040) \\
& +\mathcal{D}_{2}(0+0.029) \\
& \left.+\mathcal{D}_{3}(0+0.092)\right\}
\end{aligned}
$$

Since $1 \geq z=\tau_{H} / \tau \geq \tau_{H}$, the SV terms are certainly the dominant contributions to the cross section in the kinematic region near the hadronic threshold $\left(\tau_{H}=M_{H}^{2} / s \sim 1\right)$. At fixed $s$, this means that these terms certainly dominate in the case of heavy Higgs bosons. However, the SV terms can give the dominant effect even long before the threshold region in the hadronic cross section is actually approached [30]. This is because, in the evaluation of the hadronic cross section in Eq. (3), the partonic cross section $\hat{\sigma}_{a b}\left(\hat{s}, M_{H}^{2}\right)$ has to be weighted with the parton luminosities, which are strongly suppressed at large $\tau=\hat{s} / s=x_{1} x_{2}$. In other words, owing to the strong 
suppression of the parton densities $f_{a}\left(x, \mu_{F}^{2}\right)$ at large $x$, the partonic centre-of-mass energy $\sqrt{\hat{s}}$ is typically substantially smaller than $\sqrt{s}\left(\langle\hat{s}\rangle=\left\langle x_{1} x_{2} s\right\rangle=\langle\tau\rangle s\right)$, and the variable $z=M_{H}^{2} / \hat{s}$ in $G_{a b}(z)$ can be close to unity also when $\sqrt{s}$ is not very close to $M_{H}$.

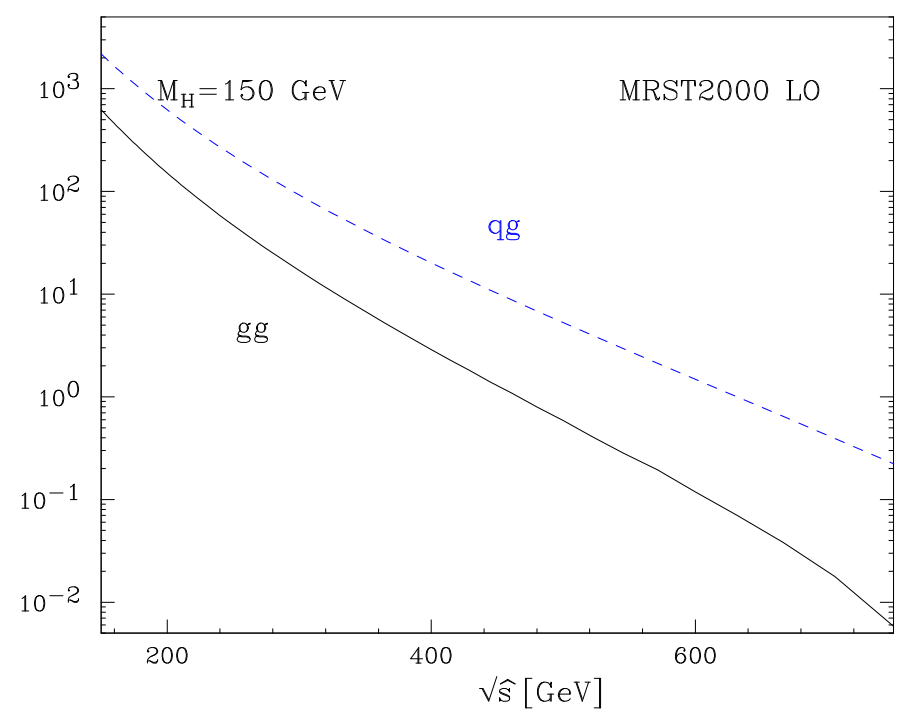

Figure 1: Parton (gg and $q g+g \bar{q}$ ) luminosities $\mathcal{L}\left(\tau, \mu_{F}^{2}\right)$ with $\mu_{F}=M_{H}=150$ GeV at the Tevatron Run II as a function of the partonic centre-of-mass energy $\sqrt{\hat{s}}(\hat{s}=\tau s)$. We use the LO set of parton distributions in Ref. 24].

At fixed $M_{H}$, the quantitative reliability of the large- $z$ approximation depends on the value of $\sqrt{s}$ and on the actual value of the parton luminosities $\mathcal{L}_{a b / h_{1} h_{2}}\left(\tau, \mu_{F}^{2}\right)$ in Eq. (3). In Fig. 11 the $g g$ and $(q g+g \bar{q})$ luminosities (with $\mu_{F}=M_{H}=150 \mathrm{GeV}$ ) at the Tevatron Run II are plotted as a function of the partonic centre-of-mass energy $\sqrt{\hat{s}}(\hat{s}=\tau s)$. We see that the luminosities decrease by almost two orders of magnitude when $\sqrt{\hat{s}}$ increases from $M_{H}=150 \mathrm{GeV}$ (hadronic threshold) to $300 \mathrm{GeV}$. A similar effect occurs at the LHC (see Fig. 1 in Ref. [26]). We thus expect that the large- $z$ expansion of the coefficient function $G_{a b}(z)$ reliably approximates the size of the higher-order QCD corrections to Higgs boson production at the Tevatron and the LHC. At NLO, this was explicitly checked in Ref. [15], by comparison with the complete expression of $G_{a b}^{(1)}(z)$.

The authors of Ref. [15] also pointed out that at NLO the numerical effect of the logarithmic term $\ln (1-z)$ of collinear origin is not small. Following this observation, in Ref. [16] we introduced the soft-virtual-collinear (SVC) approximation of the coefficient function $G_{a b}(z)$. The SVC approximation is defined by including the leading $\ln ^{k}(1-z)$ contribution from the collinear region in the $g g$ channel:

$$
\begin{aligned}
& G_{g g}^{(1) \operatorname{SVC}}\left(z ; M_{H}^{2} / \mu_{R}^{2} ; M_{H}^{2} / \mu_{F}^{2}\right)=G_{g g}^{(1) \mathrm{SV}}\left(z ; M_{H}^{2} / \mu_{R}^{2} ; M_{H}^{2} / \mu_{F}^{2}\right)-12 \ln (1-z) \\
& G_{g g}^{(2) \operatorname{SVC}}\left(z ; M_{H}^{2} / \mu_{R}^{2} ; M_{H}^{2} / \mu_{F}^{2}\right)=G_{g g}^{(2) \mathrm{SV}}\left(z ; M_{H}^{2} / \mu_{R}^{2} ; M_{H}^{2} / \mu_{F}^{2}\right)-72 \ln ^{3}(1-z) .
\end{aligned}
$$

Since the term $\ln ^{k}(1-z)$ added to the SV expressions is that with the highest power $k$ at each perturbative order ( $k=1$ and $k=3$ at LO and NLO, respectively), the SVC approximation consistently includes the next-to-dominant contribution to $G_{g g}^{(2)}$ in the limit $z \rightarrow 1$. The comparison 
between the SV and the SVC approximations can thus be used [16 to gauge the quantitative accuracy of approximating $G_{a b}(z)$ by its large- $z$ limit. In the next section we study the impact of the SV and SVC approximations at the Tevatron Run II.

Two different large- $z$ approximations, named 'soft' and 'soft+sl' were also considered in the numerical study of Ref. [17]. The 'soft' approximation of Ref. [17] regards the whole partonic cross section $\hat{\sigma}_{a b}$ in Eq. (2), while our SV approximation refers only to the hard coefficient function $G_{a b}(z)$. In other words, we perform the soft approximation on the phase-space integral of the matrix elements squared, while the kinematical flux factor $1 / \hat{s}$ in $\hat{\sigma}_{a b}$ is kept fixed and not expanded around $\hat{s}=s$. This means that we expand $G_{a b}(z)$ around $z=1$, whereas the authors of Ref. [17] consider the expansion of $\widetilde{G}_{a b}(z)=z G_{a b}(z)$. Owing to the identity

$$
z \mathcal{D}_{i}=\mathcal{D}_{i}-\ln ^{i}(1-z)
$$

the two expansions differ by subdominant $\ln ^{i}(1-z)$ contributions of kinematical origin (see also Sect. 3 in Ref. [16]). Moreover, the 'soft + sl' approximation of $\widetilde{G}_{g g}^{(2)}$ 17 includes also additional subleading terms, proportional to $\ln ^{2}(1-z)$ and $\ln (1-z)$, whose coefficients (unlike those in Eqs. (11) and (13)) are not (exactly) predictable at present [15. In summary, we note that there is no one-to-one correspondence between 'soft' (or 'soft+sl') in Ref. [17 and SV (or SVC) in our definition, the difference being due to logarithmic contributions that, formally, are consistently subdominant when $z \rightarrow 1$.

Although, from a formal viewpoint, it is legitimate to perform the large- $z$ expansion of either $G_{a b}(z)$ or $\widetilde{G}_{a b}(z)$, the two expansions can quantitatively differ when applied to the evaluation of the hadronic cross section in Eq. (11). In fact, when using $\widetilde{G}_{a b}(z)$, the analogue of Eq. (3) is

$$
\sigma\left(s, M_{H}^{2}\right)=\sigma_{0} \sum_{a, b} \int_{\tau_{H}}^{1} \frac{d \tau}{\tau} \widetilde{\mathcal{L}}_{a b / h_{1} h_{2}}\left(\tau, \mu_{F}^{2}\right) \widetilde{G}_{a b}\left(\tau_{H} / \tau\right)
$$

Comparing Eqs. (3) and (15), we see that the coefficient function $\widetilde{G}_{a b}\left(z=\tau_{H} / \tau\right)$ is convoluted with the momentum-fraction luminosity $\widetilde{\mathcal{L}}\left(\tau, \mu_{F}^{2}\right)=\tau \mathcal{L}\left(\tau, \mu_{F}^{2}\right)$. Owing to the relative rescaling factor $\tau=\hat{s} / s, \widetilde{\mathcal{L}}\left(\tau, \mu_{F}^{2}\right)$ is much less steep than the luminosity $\mathcal{L}\left(\tau, \mu_{F}^{2}\right)$ (see Fig. 11) that enters Eq. (3). Therefore, in the numerical evaluation of the Higgs boson cross section, we expect and anticipate (see the comment at the end of Sect. 3) that the large- $z$ expansion of $\widetilde{G}_{a b}(z)$ [15, 17] converges more slowly than that of $G_{a b}(z)$. The slowing down is ultimately caused by a too extreme kinematics approximation of the partonic cross section in Eq. (2): the flux factor 1/ $\hat{s}$ has been replaced by $1 / M_{H}^{2}$.

\section{Inclusive production at the Tevatron Run II}

In this section we study the higher-order QCD corrections to the inclusive production of the SM Higgs boson at the Tevatron Run II, i.e. proton-antiproton collisions at $\sqrt{s}=2 \mathrm{TeV}$. We recall that we include the exact dependence on $M_{\text {top }}$ in the Born-level cross section $\sigma_{0}$ (see Eq. (5)), while the coefficient function $G_{a b}(z)$ is evaluated in the large- $M_{\text {top }}$ limit. At NLO [14, 15] this is a very good numerical approximation when $M_{H} \leq 2 M_{\text {top }}$. 
Unless otherwise stated, cross sections are computed using the MRST2000 24] sets of parton distributions with densities and coupling constant evaluated at each corresponding order, i.e. using LO distributions and 1-loop $\alpha_{\mathrm{S}}$ for the LO cross section, and so forth. The corresponding values of $\Lambda_{Q C D}^{(5)}\left(\alpha_{\mathrm{S}}\left(M_{Z}\right)\right)$ are $0.132(0.1253), 0.22(0.1175)$ and $0.187(0.1161) \mathrm{GeV}$, at 1-loop, 2-loop and 3-loop order, respectively. In the NNLO case we use the 'central' set of MRST2000, obtained from a global fit of data (deep inelastic scattering, Drell-Yan production and jet $E_{T}$ distribution) by using the approximate NNLO evolution kernels presented in Ref. [25]. The result we refer to as NNLO-SV(SVC) corresponds to the sum of the LO and exact NLO (including the $q g$ and $q \bar{q}$ channels) contributions plus the SV(SVC) corrections at NNLO, given in Eq. (11) (Eq. (13)).

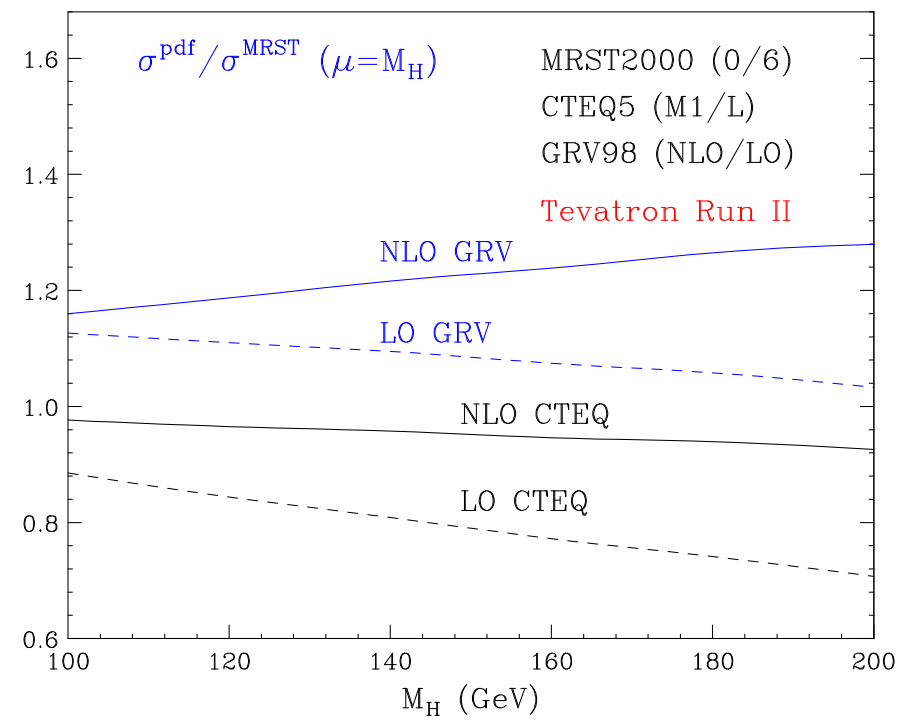

Figure 2: Relative difference of LO and NLO results computed with GRV98 and CTEQ5 distributions with respect to MRST2000 distribution.

In Fig. 2 we show the dependence of the LO and NLO cross sections on the choice of parton distributions. The results obtained by using both the GRV98 [31] and CTEQ5 [32] sets differ substantially from the MRST2000 results. In the case of the GRV98 sets, this difference is not unexpected, since the value of $\alpha_{\mathrm{S}}\left(M_{Z}\right)$ and the gluon distribution are quite different from those of MRST2000 and CTEQ5. We see that, as $M_{H}$ increases from 100 to $200 \mathrm{GeV}$, the LO result obtained by using the CTEQ5 set is between 10 and 30\% lower than the one obtained by MRST2000. At NLO the difference is smaller, and it is compatible with the $\pm 10 \%$ uncertainty recommended by the CTEQ collaboration [33] on $g g$ and $q g$ luminosities in the $x$ region that controls Higgs boson production at the Tevatron. Although the LO differences are physically less meaningful than the NLO differences, the size of the former has to be kept in mind when quoting QCD predictions (based on either analytic calculations or Monte Carlo event generators) that directly or indirectly (e.g. in the case of $K$-factors) use LO parton densities.

Figure 3 shows the scale dependence of the cross section for the production of a Higgs boson with $M_{H}=150 \mathrm{GeV}$. The scale dependence is analysed by varying the factorization and renormalization scales by a factor of $4 \mathrm{up}$ and down from the central value $M_{H}$. The plot on the left corresponds to the simultaneous variation of both scales, $\mu_{F}=\mu_{R}=\chi M_{H}$, whereas the plots in the centre and on the right respectively correspond to the results of the independent variation of 


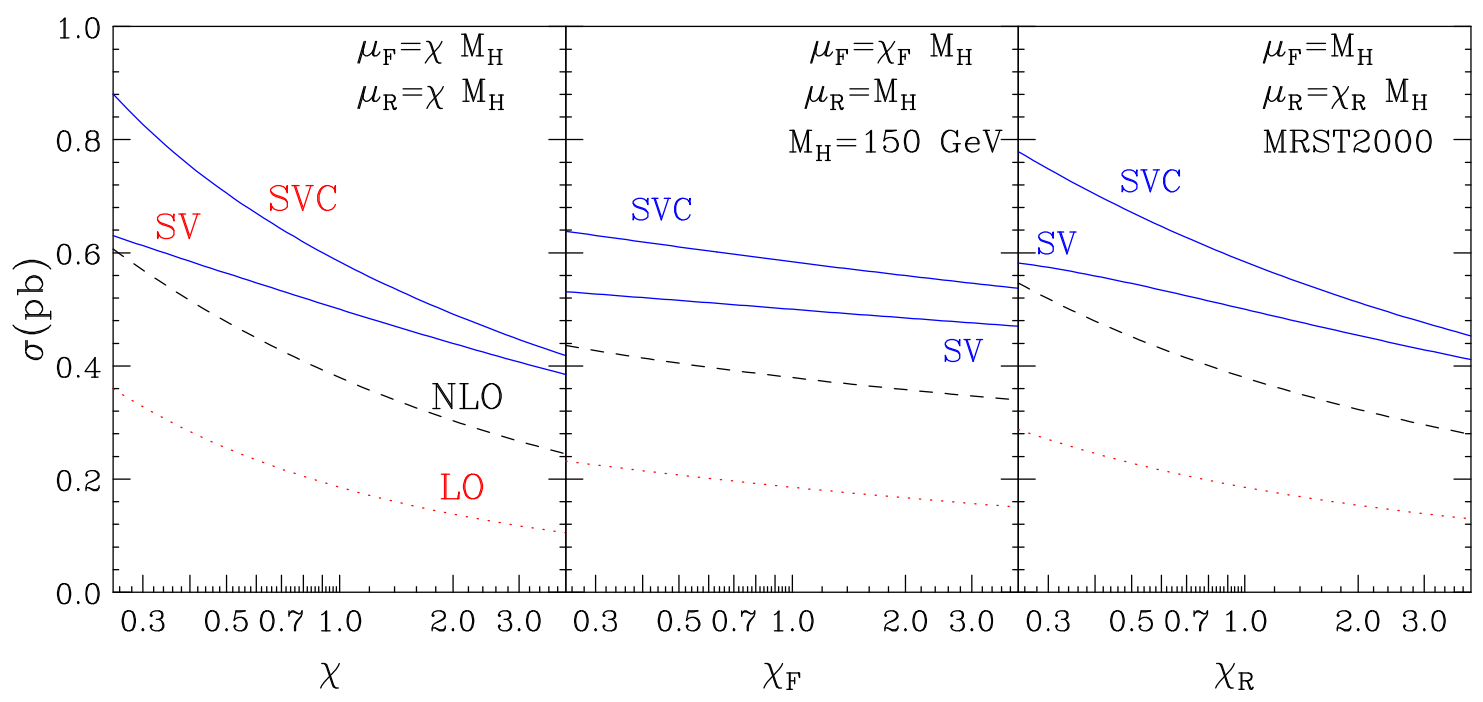

Figure 3: Scale dependence of the Higgs production cross section for $M_{H}=150$ GeV at LO, NLO, $N N L O-S V$ and $N N L O-S V C$.

the factorization and renormalization scales, keeping the other fixed at the central value.

As expected from the QCD running of $\alpha_{\mathrm{S}}$, the cross sections typically decrease when $\mu_{R}$ increases around the characteristic hard scale $M_{H}$. A similar behaviour is observed when $\mu_{F}$ varies, since the cross sections are mainly sensitive to partons with momentum fraction $x \sim 0.05-$ 0.1 , and in this $x$ range scaling violation of the parton densities is (slightly) negative. The largest variations in the cross section calculation are thus obtained by simultaneously varying $\mu_{R}$ and $\mu_{F}$. The scale dependence is mostly driven by the renormalization scale, because the lowest-order contribution to the process is proportional to $\alpha_{\mathrm{S}}^{2}$, a (relatively) high power of $\alpha_{\mathrm{S}}$.

In summary, Fig. 3 shows that the scale dependence is reduced when higher-order corrections are included. Varying the scales in the range $0.5 \leq \chi_{R}, \chi_{F} \leq 2$, the reduction is from about $\pm 20 \%$ at full NLO to about $\pm 10 \%$ and $\pm 15 \%$ at NNLO-SV and NNLO-SVC, respectively. The increase in the scale dependence when going from NNLO-SV to NNLO-SVC is due to the fact that the contribution of the dominant collinear terms included in the SVC approximation (see Eqs. (12) and (13)) is not small and scale-independent, so it cannot be compensated by scale variations.

In Fig. 1 we study the $K$-factors, defined as the ratio of the cross section evaluated at each corresponding order over the LO result. Since the LO result sizeably depends on the choice of parton distributions, the $K$-factor should be interpreted with care. For instance, for $M_{H}=$ $180 \mathrm{GeV}$, the NLO $K$-factor computed by using MRST distributions is about $20 \%$ smaller than the one obtained by using CTEQ distributions.

In Fig. 1t the bands account for the 'theoretical uncertainty' due to the scale dependence, quantified by using the minimum and maximum values of the cross sections when the scales $\mu_{R}$ and $\mu_{F}$ are varied (simultaneously and independently, as in Fig. 3) in the range $0.5 \leq \chi_{R}, \chi_{F} \leq 2$. The LO result that normalizes the $K$-factors is computed at the default scale $M_{H}$ in all cases.

\footnotetext{
${ }^{\S}$ At the LHC, the $\mu_{F}$ dependence is opposite, as shown in Fig. 1 of Ref. [16].
} 


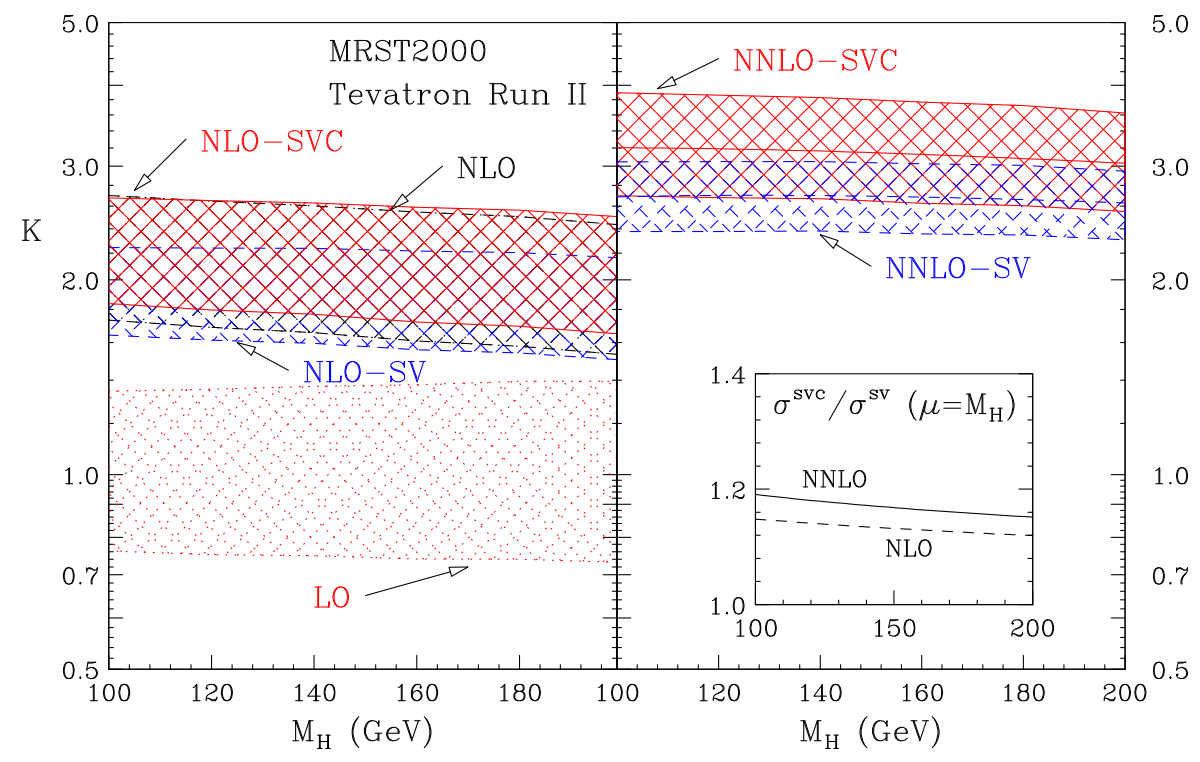

Figure 4: K-factors for Higgs production for the full $N L O$ result and the $N L O-S V$, NLO-SVC, NNLO-SV and NNLO-SVC approximations.

The plot on the left-hand side of Fig. 1 shows the scale uncertainty at LO and compares the full NLO result with the NLO-SV and NLO-SVC approximations. When $M_{H}$ is in the range 140-180 GeV, the NLO-SV approximation tends to underestimate the full NLO result by about 7-8\%, whereas the NLO-SVC approximation overestimates it by about $4-5 \%$, showing the effect of the term $\ln (1-z)$ added in the SVC approximation. We find that the SVC result provides an excellent approximation of the full $g g$ contribution at NLO, the difference being less than 2 per mille. The contribution of the $q g$ channel tends to lower the SVC result by $\lesssim 5 \%$. The effect of the $q g$ channel is mainly due to the logarithmically-enhanced behaviour $G_{q g}^{(1)}(z) \simeq \frac{4}{3} \ln (1-z)$ of the corresponding coefficient function at large $z$. The improved reliability of the SV and SVC approximations with respect to the LHC [16] is not unexpected since at the Tevatron we are closer to threshold.

The right-hand side of Fig. 1 shows the SV and SVC results at NNLO. Again, the SVC band sits higher than the SV one and, as shown in the inset plot, the ratio of the corresponding cross sections increases with respect to NLO. The contribution from non-leading terms $\ln ^{k}(1-z)$, with $k<3$ (which are not under control within the SVC approximation), is not included. We have tried to add a term $\ln ^{2}(1-z)$ with a coefficient as large as the one of the $\ln ^{3}(1-z)$ contribution, and we have found a small $(\mathcal{O}(5 \%))$ modification. Therefore, we expect the effect of these nonleading logarithmic terms to be numerically less important. As pointed out in Ref. [16], at NNLO there is a (still unknown) leading collinear contribution proportional to $\ln ^{3}(1-z)$ also in the $q g$ channel. Owing to the size of the contribution of the $q g$ channel at NLO, the quantitative effect of this NNLO term can be of the same order as the corresponding one included through the SVC approximation in the $g g$ channel.

We recall that the NNLO results on the right-hand side of Fig. 1 are obtained by using the (approximated) NNLO parton distributions of the MRST2000 set. Using the NLO parton distributions, the $K$-factors would be smaller by about 5 to $8 \%$ as $M_{H}$ increases from 120 to $180 \mathrm{GeV}$. 


\begin{tabular}{|c|c|c|c|c|c|c|}
\hline$M_{H}(\mathrm{GeV})$ & LO & NLO & NLO-SV & NLO-SVC & NNLO-SV & NNLO-SVC \\
\hline 140 & 0.2282 & 0.4715 & 0.4338 & 0.4922 & 0.6163 & 0.7222 \\
\hline 150 & 0.1856 & 0.3794 & 0.3507 & 0.3969 & 0.5002 & 0.5841 \\
\hline 160 & 0.1523 & 0.3080 & 0.2859 & 0.3229 & 0.4092 & 0.4763 \\
\hline 170 & 0.1260 & 0.2519 & 0.2349 & 0.2646 & 0.3369 & 0.3912 \\
\hline 180 & 0.1050 & 0.2075 & 0.1943 & 0.2184 & 0.2795 & 0.3235 \\
\hline
\end{tabular}

Table 1: Cross sections in pb as a function of $M_{H}$. The calculation is performed by setting $\mu_{R}=\mu_{F}=M_{H}$.

In summary, considering the results obtained at NLO, we expect the full NNLO $K$-factor to be between the SV and SVC bands. For reference, we give in Table 1 the central values of the cross section in the range $M_{H}=140-180 \mathrm{GeV}$. In particular, for a light Higgs boson $\left(M_{H} \lesssim 200 \mathrm{GeV}\right)$, this corresponds to an increase of about $50 \%$ with respect to the full NLO result, i.e. a factor of $\sim 3$ with respect to the LO result. Taking into account that the NLO result increases the LO cross section by a factor of about 2, our result shows that the convergence of the perturbative series is poorer at the Tevatron than at the LHC [16]. This also implies that QCD contributions beyond NNLO can still be significant at the Tevatron.

Our conclusion on the possible relevance of higher-order contributions is not in contradiction with the improved scale dependence of the NNLO calculation. As is well known, the customary procedure of varying the scales to estimate the theoretical uncertainty due to missing higher-order terms can only give a lower limit on the 'true' uncertainty. This is well demonstrated by the plot on the left-hand side of Fig. 1 , which shows no overlap between the LO and NLO bands. Since the NLO and NNLO bands still tend to show no (or a marginal) overlap, their size cannot yet be regarded as a reliable estimate of the theoretical uncertainty.

A comment on the numerical results presented in Ref. [17] is in order. The authors of Ref. [17] use different parton distributions and, as explained in Sect. 2, there is no one-to-one correspondence between their 'soft' (or 'soft+sl') approximation and our SV (or SVC) approximation. Therefore, those results cannot directly be compared with ours (see also Ref. [34]). Certainly, they show a slower numerical convergence of the large- $z$ expansion used in Ref. [17]. As discussed at the end of Sect. 2, this is not unexpected because of significant subdominant effects of kinematical origin.

\section{Vetoed cross section}

Direct Higgs production followed by the decay $H \rightarrow W W \rightarrow l^{+} l^{-} \nu \bar{\nu}$ is an important channel to discover the SM Higgs boson in the intermediate-mass range $140 \mathrm{GeV} \lesssim M_{H} \lesssim 190 \mathrm{GeV}$ both at the Tevatron and at the LHC. Nevertheless, as recalled in Sect. 1, several experimental cuts have to be applied to discriminate the signal over the background. An important selection to enhance the statistical significance is a veto on the high transverse-momentum jets that accompany the production of the Higgs boson. Events with high transverse-momentum jets are excluded from the analysis [6, 17, 11].

In this section we study the effect of a jet veto on inclusive Higgs production. The events that 
pass the veto selection are those with $p_{T}^{\text {jet }}<p_{T}^{\text {veto }}$, where $p_{T}^{\text {jet }}$ is the transverse momentum of any final-state jets. Jets are defined by a cone algorithm. In the perturbative calculation, jets are represented by a parton or a set of partons. The Higgs boson can be accompanied by one finalstate parton at NLO, and by one or two final-state partons at NNLO. In the case of a single finalstate parton with transverse momentum $\mathbf{p}_{1 T}$, the vetoed cross section is computed by imposing $\left|\mathbf{p}_{1 T}\right|<p_{T}^{\text {veto }}$ (i.e. we veto events with $\left|\mathbf{p}_{1 T}\right|>p_{T}^{\text {veto }}$ ). When there are two final-state partons with transverse momenta $\mathbf{p}_{1 T}$ and $\mathbf{p}_{2 T}$, we consider their angular distance $R_{12}^{2}=\left(\eta_{1}-\eta_{2}\right)^{2}+\left(\phi_{1}-\phi_{2}\right)^{2}$ in the pseudorapidity-azimuth plane: if $R_{12}>R$, we impose the constraints $\left|\mathbf{p}_{1 T}\right|,\left|\mathbf{p}_{2 T}\right|<p_{T}^{\text {veto }}$ (i.e. we veto events with $\left|\mathbf{p}_{1 T}\right|>p_{T}^{\text {veto }}$ or $\left|\mathbf{p}_{2 T}\right|>p_{T}^{\text {veto }}$ ); if $R_{12}<R$, we combine the two partons in a single jet and we impose $\left|\mathbf{p}_{1 T}+\mathbf{p}_{2 T}\right|<p_{T}^{\text {veto }}$ (i.e. we veto events with $\left|\mathbf{p}_{1 T}+\mathbf{p}_{2 T}\right|>p_{T}^{\text {veto }}$ ). In all the numerical results presented in this section, the cone size $R$ of the jets is fixed at the value $R=0.4$.

As in the case of the inclusive cross section, we evaluate the jet-vetoed cross section by using the large- $M_{\text {top }}$ limit. Studies on Higgs + jets production show that the QCD matrix elements in the large- $M_{\text {top }}$ limit [35, 36] approximate very well those with the exact $M_{\text {top }}$ dependence [37, 38], provided the jet transverse momenta and, hence, $p_{T}^{\text {veto }}$ remain small with respect to $M_{\text {top }}$.

The vetoed cross section $\sigma^{\text {veto }}\left(s, M_{H}^{2} ; p_{T}^{\text {veto }}, R\right)$ can be written as

$$
\sigma^{\text {veto }}\left(s, M_{H}^{2} ; p_{T}^{\text {veto }}, R\right)=\sigma\left(s, M_{H}^{2}\right)-\Delta \sigma\left(s, M_{H}^{2} ; p_{T}^{\text {veto }}, R\right)
$$

where $\sigma\left(s, M_{H}^{2}\right)$ is the inclusive hadronic cross section in Eq. (1), and $\Delta \sigma$ is the 'loss' in cross section due to the jet-veto procedure. The vetoed cross section is computable by a factorization formula analogous to Eq. (11), apart from the replacement of the coefficient function $G_{a b}(z)$ by the vetoed coefficient function $G_{a b}^{\mathrm{veto}}\left(z ; \pi_{T}, R\right)$. By analogy with Eq. (16), we can write the vetoed coefficient function as

$$
G_{a b}^{\mathrm{veto}}\left(z ; \pi_{T}, R\right)=G_{a b}(z)-\Delta G_{a b}\left(z ; \pi_{T}, R\right)
$$

where, to simplify the notation (see Eq. (20)), the dependence on $p_{T}^{\text {veto }}$ is parametrized by the dimensionless variable $\pi_{T}$ :

$$
\pi_{T}\left(z, p_{T}^{\mathrm{veto}} / M_{H}\right) \equiv \frac{2 p_{T}^{\mathrm{veto}} \sqrt{z}}{(1-z) M_{H}}
$$

At LO, the vetoed cross section is equal to the inclusive one, so the subtracted coefficient function $\Delta G_{a b}$ vanishes. At higher perturbative orders, $\Delta G_{a b}$ is computable according to the power-series expansion

$$
\begin{aligned}
\Delta G_{a b}\left(z ; \pi_{T}, R ; \alpha_{\mathrm{S}}\left(\mu_{R}^{2}\right), M_{H}^{2} / \mu_{R}^{2} ; M_{H}^{2} / \mu_{F}^{2}\right) & =\frac{\alpha_{\mathrm{S}}^{3}\left(\mu_{R}^{2}\right)}{\pi} \Delta G_{a b}^{(1)}\left(z ; \pi_{T}\right) \Theta\left(1-\pi_{T}\left(z, p_{T}^{\text {veto }} / M_{H}\right)\right) \\
& +\frac{\alpha_{\mathrm{S}}^{4}\left(\mu_{R}^{2}\right)}{\pi^{2}} \Delta G_{a b}^{(2)}\left(z ; \pi_{T}, R ; \frac{M_{H}^{2}}{\mu_{R}^{2}} ; \frac{M_{H}^{2}}{\mu_{F}^{2}}\right)+\mathcal{O}\left(\alpha_{\mathrm{S}}^{5}\right) .
\end{aligned}
$$

The NLO contribution $\Delta G_{a b}^{(1)}$ is scale-independent and $R$-independent, but it depends on $p_{T}^{\text {veto }}$. 
We have explicitly evaluated it in the large- $M_{\text {top }}$ limit, and we find:

$$
\begin{aligned}
& \Delta G_{g g}^{(1)}\left(z ; \pi_{T}\right)=\hat{P}_{g g}(z) \ln \frac{1+\sqrt{1-\pi_{T}^{2}}}{1-\sqrt{1-\pi_{T}^{2}}}-\frac{11}{2} \frac{(1-z)^{3}}{z}\left(1-\frac{\pi_{T}^{2}}{22}\right) \sqrt{1-\pi_{T}^{2}} \\
& \Delta G_{g q}^{(1)}\left(z ; \pi_{T}\right)=\frac{1}{2} \hat{P}_{g q}(z) \ln \frac{1+\sqrt{1-\pi_{T}^{2}}}{1-\sqrt{1-\pi_{T}^{2}}}-\frac{(1-z)^{2}}{z} \sqrt{1-\pi_{T}^{2}} \\
& \Delta G_{q \bar{q}}^{(1)}\left(z ; \pi_{T}\right)=\frac{32}{27} \frac{(1-z)^{3}}{z}\left(1-\frac{\pi_{T}^{2}}{4}\right) \sqrt{1-\pi_{T}^{2}}
\end{aligned}
$$

where

$$
\begin{aligned}
& \hat{P}_{g q}(z)=\frac{4}{3} \frac{1+(1-z)^{2}}{z} \\
& \hat{P}_{g g}(z)=6\left[\frac{1-z}{z}+\frac{z}{1-z}+z(1-z)\right] .
\end{aligned}
$$

The evaluation of the NNLO contribution $\Delta G_{a b}^{(2)}$ cannot be easily performed in analytic form, since the calculation depends on the details of the jet algorithm. We compute $\Delta G_{a b}^{(2)}$ numerically by using the program of Ref. [27]. The program, which implements the matrix elements (in the large- $M_{\text {top }}$ limit) of Refs. [35, 36] by using the subtraction-method procedure of Ref. [39], computes the QCD corrections to Higgs+jet(s) production up to order $\alpha_{\mathrm{S}}^{4}$. For the purpose of evaluating the contribution $\Delta \sigma$ to Eq. (16), we use the program to compute the Higgs + jet(s) cross section when the transverse momentum of the highest- $p_{T}$ jet is larger than $p_{T}^{\text {veto }}$.

In the following we present both NLO and NNLO numerical results for the vetoed cross section $\sigma^{\text {veto }}$ in Eq. (16). The results are obtained by using the parton distributions of the MRST2000 set, as explained in Sect. 3. The NLO calculation is exact: apart from using the large- $M_{\text {top }}$ limit, we do not perform any further approximations. At the NNLO, the contribution $\Delta \sigma$ to Eq. (16) is again evaluated exactly, while to evaluate the contribution of the inclusive cross section we rely on our approximate estimate in Sect. 3 (see the corresponding LHC results in Sect. 4 of Ref. [16]) and, in particular, we use the NNLO-SVC result. Therefore, once the full NNLO result for the inclusive cross section is available, it can straightforwardly be used to 'correct' our NNLO estimate for the vetoed cross section. As stated above, in our numerical calculations we fixed the cone size of the jets to the value $R=0.4$. The $R$-dependence of the perturbative calculation first appears at the NNLO. In particular, the NNLO vetoed cross section decreases by increasing $R$.

Note that the numerical program of Ref. [27] is a Monte Carlo code that evaluates Higgs+jet(s) production at the fully exclusive level. Therefore, it can be used through the subtraction procedure of Eq. (16) to compute vetoed cross sections also when additional kinematical cuts (e.g. cuts on the rapidities of the jets, or asymmetric cuts on the jet transverse momenta) or different jet definitions are considered.

We first present the vetoed cross section at the Tevatron Run II. In Fig. 5 we show the dependence of the NLO results on the Higgs mass for different values of $p_{T}^{\text {veto }}(15,20,30$ and

IThe numerical program of Ref. [27] implements the large- $M_{\text {top }}$ limit strictly, i.e. also the Born-level contribution $\sigma_{0}$ in Eq. (5) is evaluated in the limit $M_{H} / M_{\text {top }} \rightarrow 0$. For simplicity, the numerical results of this section implement the same approximation. The approximation used in Sect. 3 can be recovered by simply rescaling the absolute value of the cross sections by the overall factor $\sigma_{0} / \sigma_{0}\left(M_{H} / M_{\mathrm{top}}=0\right)$. Of course, such a rescaling has no effects on the ratios $\Delta \sigma / \sigma$ and on the vetoed $K$-factors. 

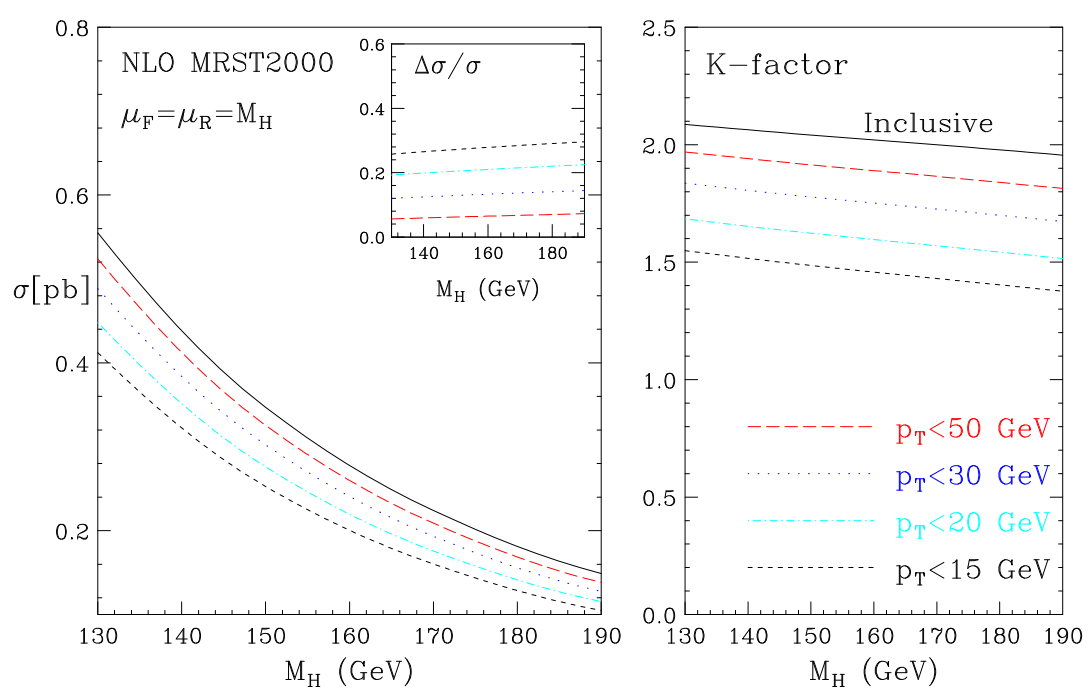

Figure 5: Vetoed cross section and K-factors: NLO results at the Tevatron Run II.

$50 \mathrm{GeV})$. The vetoed cross sections $\sigma^{\text {veto }}\left(s, M_{H}^{2} ; p_{T}^{\text {veto }}, R\right)$ and the inclusive cross section $\sigma\left(s, M_{H}^{2}\right)$ are given in the plot on the left-hand side. The inset plot gives an idea of the 'loss' in cross section once the veto is applied, by showing the ratio between the cross section difference $\Delta \sigma$ in Eq. (16) and the inclusive cross section at the same perturbative order. As can be observed, for large values of the cut, say $p_{T}^{\text {veto }}=50 \mathrm{GeV}$, less than $10 \%$ of the inclusive cross section is vetoed. The veto effect increases by decreasing $p_{T}^{\text {veto }}$, but it is still smaller than $30 \%$ when $p_{T}^{\text {veto }}=15 \mathrm{GeV}$. On the right-hand side of Fig. 占, we show the corresponding $K$-factors, i.e. the vetoed cross sections normalized to the $\mathrm{LO}$ result, which is independent of the value of the cut.
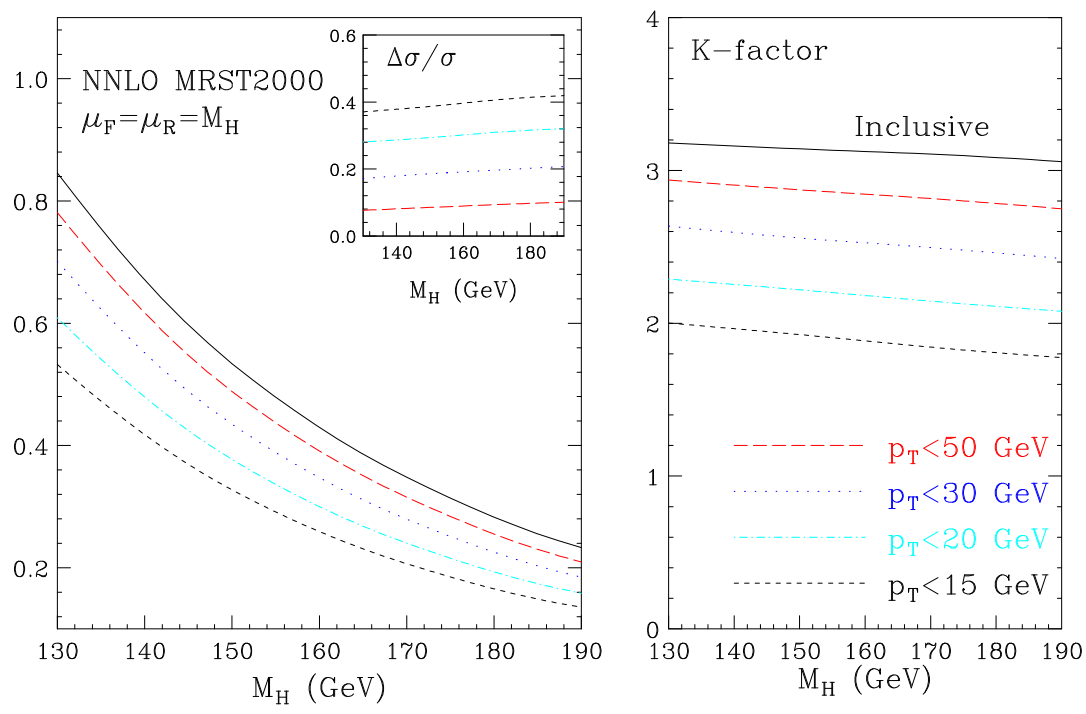

Figure 6: Vetoed cross section and K-factors: NNLO results at the Tevatron Run II.

Figure 6 shows the analogous results at NNLO. Note that, although $\Delta \sigma / \sigma$ is slightly larger than at NLO, the vetoed cross sections and, thus, the $K$-factors, are still larger than at NLO. This 
is mostly due to the large increase of the inclusive cross section at NNLO, as shown in Sect. 3 .

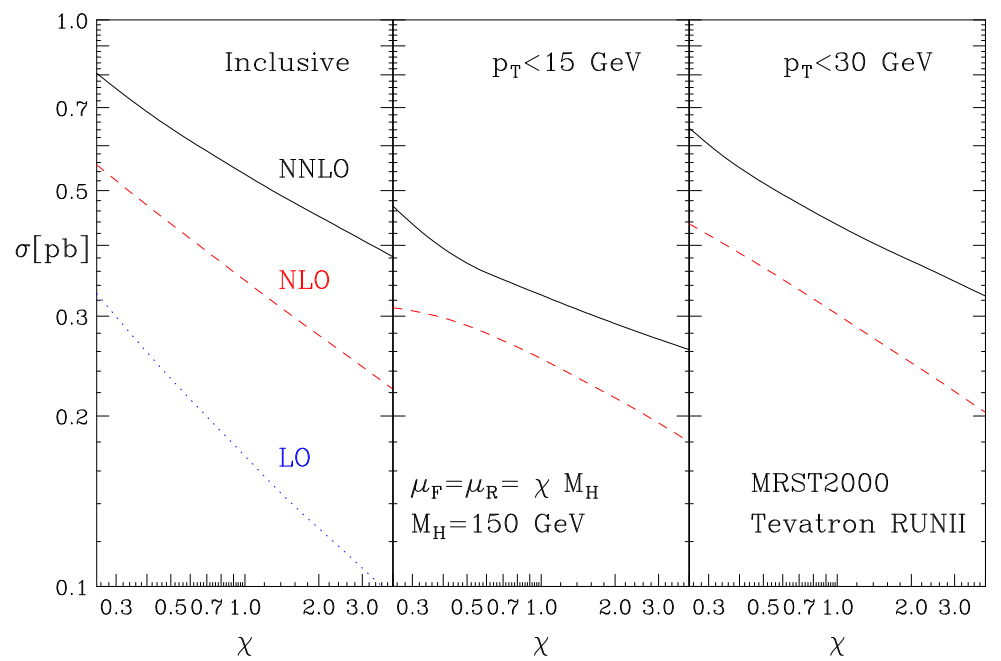

Figure 7: Scale dependence of the inclusive and vetoed cross sections at LO, NLO and NNLO-SVC at the Tevatron.

All the results plotted in Figs. 5 and 6 have been obtained by fixing the renormalization and factorization scales at the default value $\mu_{R}=\mu_{F}=M_{H}$. In Fig. 7, we study the scale dependence of the cross sections (inclusive and with $p_{T}^{\text {veto }}=15$ and $30 \mathrm{GeV}$ ) at LO, NLO and NNLO. The renormalization and factorization scales are varied simultaneously by a factor of 4 up and down with respect to $M_{H}$. No significant differences are found when the scales are varied separately. As can be observed, there is an improvement in the stability of the result when higher order corrections are included. This is particularly noticeable when going from LO to NLO, while the comparison between NNLO and NLO looks rather similar to the one observed in the inclusive case.

The scale-dependence effects on the perturbative $K$-factors can be appreciated also from Fig. 8, where the LO, NLO and NNLO-SVC bands (computed as in Fig. A) are shown in the case in which a veto of $p_{T}^{\text {veto }}=15 \mathrm{GeV}$ is applied. Comparing Fig. 8 with the inclusive case in Fig. 1 , we see that the effect of the veto is to partially reduce the relative difference between the NLO and NNLO results; the increase of the corresponding $K$-factors can be estimated to about $25 \%$.

The results on the jet veto presented so far can be qualitatively explained by a simple physical picture. As shown in Sect. 3, the effect of the higher-order contributions to inclusive Higgs boson production at the Tevatron is large. The dominant part of this effect is due to soft and collinear radiation, whereas the accompanying hard radiation has little effect. The characteristic scale of the highest transverse momentum $p_{T}^{\max }$ of the accompanying jets is indeed $p_{T}^{\max } \sim\langle 1-z\rangle M_{H}$ (see e.g. the upper bound on $p_{T}^{\text {veto }}$ from the theta function in Eq. (19)), where the average value $\langle 1-z\rangle=\left\langle 1-M_{H}^{2} / \hat{s}\right\rangle$ of the distance from the partonic threshold is small. As a consequence the jet veto procedure is weakly effective unless the value of $p_{T}^{\text {veto }}$ is pretty small (i.e. substantially smaller than $\left.p_{T}^{\max }\right)$. Decreasing $p_{T}^{\mathrm{veto}}$, the enhancement of the inclusive cross section due to soft radiation at higher orders is reduced, and the jet veto procedure tends to improve the convergence of the perturbative series (see e.g. Figs. 1 and 8). Note also that the characteristic scale $p_{T}^{\max }$ is a slightly increasing function of $M_{H}$, the linear increase with $M_{H}$ being partially compensated 


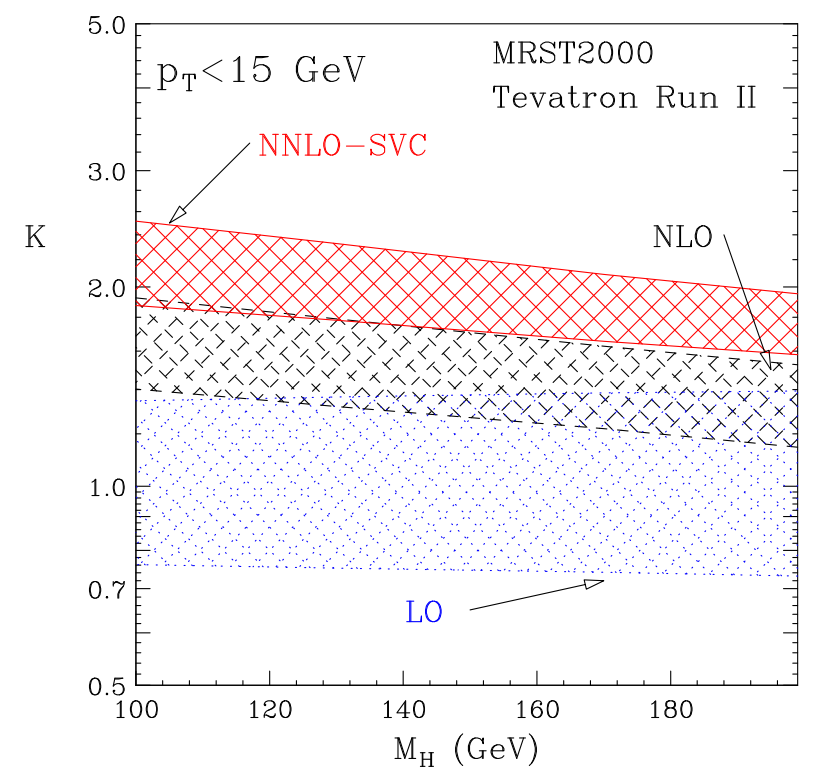

Figure 8: $K$-factors for Higgs production at the Tevatron in the case of a veto of $p_{T}^{\text {veto }}=15$ GeV: $L O, N L O$ and $N N L O-S V C$ approximation.

by the decrease of $\langle 1-z\rangle$. Therefore, at fixed $p_{T}^{\text {veto }}$ the vetoed $K$-factor decreases more than the inclusive $K$-factor when $M_{H}$ increases (see Fig. 6).

On the basis of this physical picture, we can easily anticipate the qualitative effect of the jet veto at the LHC. The overall features of the QCD corrections to inclusive Higgs boson production at the LHC [16] are the same as at the Tevatron. The main quantitative differences arise from the fact that Higgs production at the LHC is less close to threshold than at the Tevatron and, therefore, the accompanying jets are less soft $(\langle 1-z\rangle$ is larger $)$ at the LHC than at the Tevatron. At fixed $p_{T}^{\text {veto }}$ the effect of the jet veto is thus stronger at the LHC.
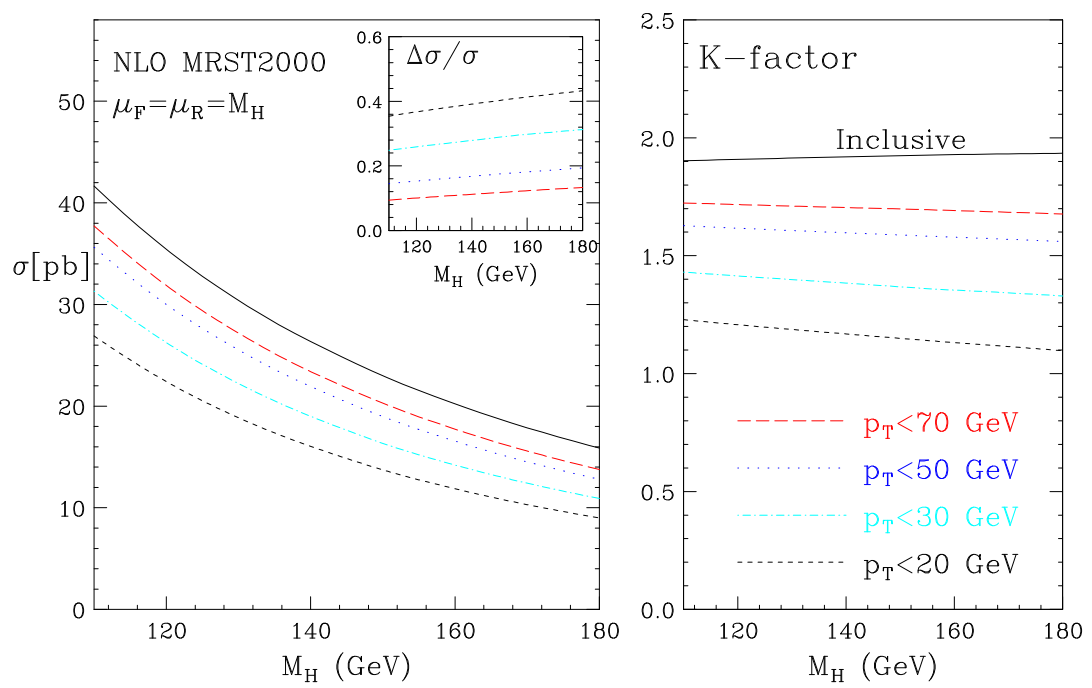

Figure 9: Vetoed cross sections and $K$-factors at NLO at the $\mathrm{LHC}$. 

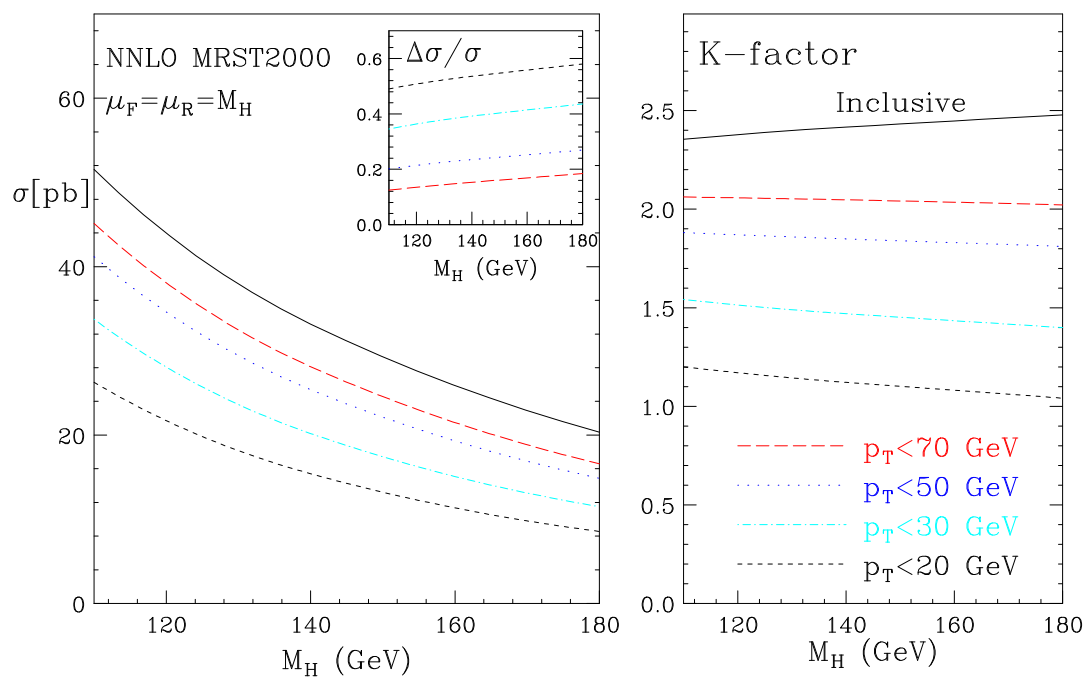

Figure 10: Vetoed cross sections and K-factors at NNLO at the LHC.

The results for the vetoed cross sections at the LHC are presented in Figs. 9 and 10 for $p_{T}^{\text {veto }}=20,30,50$ and $70 \mathrm{GeV}$. At fixed value of the cut, the impact of the jet veto, both in the 'loss' of cross section and in the reduction of the $K$-factors, is larger at the LHC than at the Tevatron Run II. This effect can also be appreciated by comparing Fig. 11 and Fig. 8. At the LHC, the value of $p_{T}^{\text {veto }}=30 \mathrm{GeV}$ is already sufficient to reduce the difference between the NNLO and NLO results to less than $10 \%$.

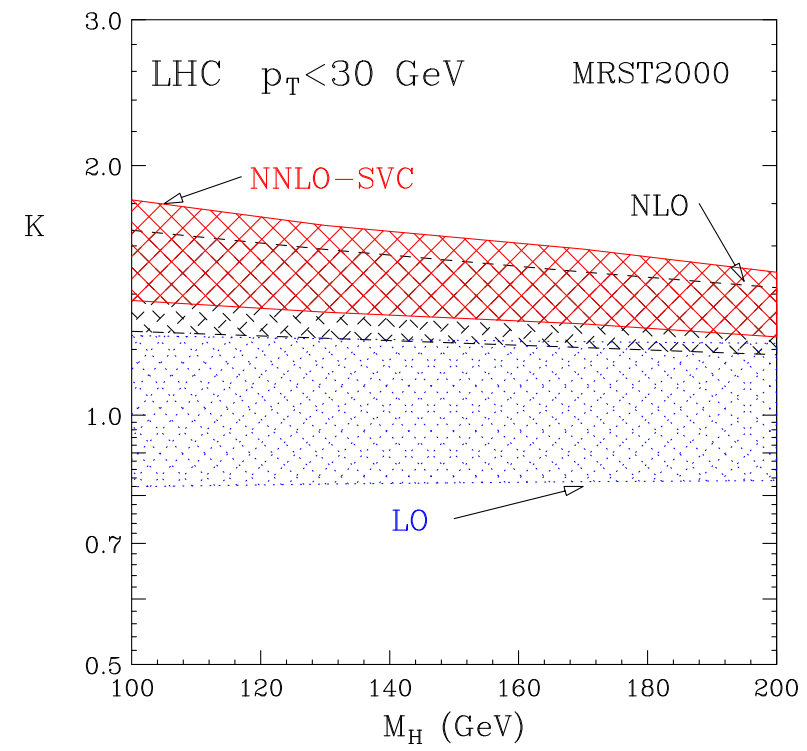

Figure 11: The same as in Fig. Q 0 , but at the LHC and with $p_{T}^{\text {cut }}=30 \mathrm{GeV}$.

Note that when $p_{T}^{\text {veto }}$ is much smaller than the characteristic scale $p_{T}^{\max } \sim\langle 1-z\rangle M_{H}$, the coefficients of the perturbative expansion of the vetoed cross section contain large logarithmically- 
enhanced contributions. For instance, from Eq. (20) we have

$$
\Delta G_{g g}^{(1)}\left(z ; \pi_{T}\right) \sim 2 \hat{P}_{g g}(z) \ln \frac{(1-z) M_{H}}{p_{T}^{\text {veto }}} .
$$

The presence of these contributions can spoil the quantitative convergence of the fixed-order expansion in $\alpha_{S}$. Since $\langle 1-z\rangle M_{H}$ is larger at the LHC than at the Tevatron, the value of $p_{T}^{\text {veto }}$ at which these effects become visible is larger at the LHC. The perturbative $K$-factors shown in Fig. 12 suggest that at the LHC the vetoed cross section is sensitive to these large logarithmic terms already when $p_{T}^{\text {veto }}=15 \mathrm{GeV}$. Indeed, the scale-dependence band is larger at NNLO than at NLO. At such small values of $p_{T}^{\text {veto, }}$, perturbative contributions beyond the NNLO can still be significant.

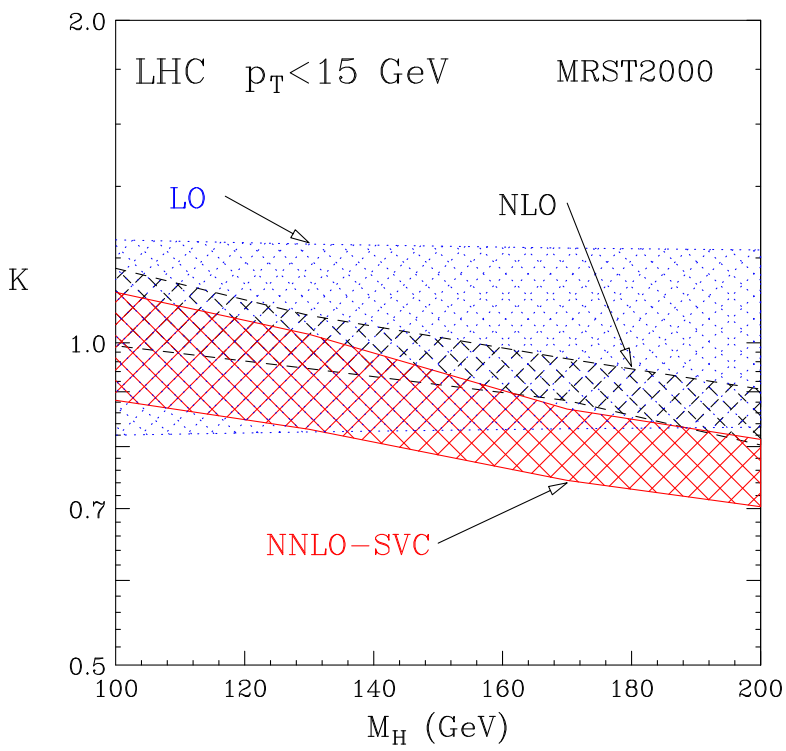

Figure 12: The same as in Fig. 8, but at the LHC and with $p_{T}^{\text {cut }}=15 \mathrm{GeV}$.

\section{Conclusions}

In this paper we have presented our study of QCD radiative corrections to direct Higgs boson production at hadron colliders and the impact of jet veto.

Using the theoretical results of Refs. [16, 17], in Sect. 3 we have first shown that the NNLO-SV and NNLO-SVC approximations are expected to be a good estimate of the full NNLO contributions to the inclusive cross section at the Tevatron Run II. Similar conclusions about the inclusive production at the LHC were obtained in Ref. [16]. The reliability of these NNLO approximations follows from the fact that a sizeable part of the higher-order QCD corrections to the partonic cross section is due to the emission of soft radiation, whereas the effect of hard radiation is suppressed by the steeply falling behaviour of the parton distributions at large $x$. As is customary practice, the perturbative expansion has been performed in the $\overline{\mathrm{MS}}$ factorization scheme. Since the $\overline{\mathrm{MS}}$-scheme 
parton densities embody (by definition) too much soft-gluon suppression (see e.g. Ref. [40]), the perturbative corrections to the partonic cross section have to compensate for it; they thus enhance the hadronic cross section. At the Tevatron, we have shown that the NNLO effect is large and increases the cross section by about $50 \%$ with respect to the NLO result. This suggest that contributions beyond the NNLO can still be significant. Since Higgs boson production is less close to threshold at the LHC than at the Tevatron, the accompanying QCD radiation is less soft at the LHC than at the Tevatron. The estimated size of the NNLO effects at the LHC [16] is thus consistently smaller than at the Tevatron.

We have then addressed the impact of a jet veto on the inclusive cross section, both at the Tevatron and at the LHC. We have presented results at NLO and NNLO. At NNLO, we have used our estimate for the inclusive cross section and we have computed (subtracted) the effect of the jet veto by using the numerical program of Ref. [27]. The jet veto reduces not only the absolute value of the cross section, but also the size of the higher-order QCD corrections. Since the accompanying QCD radiation is softer at the Tevatron than at the LHC, the impact of the veto is less effective at the Tevatron than at the LHC. In the case of a strong transverse-momentum cut of $p_{T}^{\text {veto }}=15 \mathrm{GeV}$ at the Tevatron, the NNLO contributions increase the NLO result by about $25 \%$. At the LHC, the difference between the NNLO and NLO results is already reduced to less than $10 \%$ by considering a weaker cut of $p_{T}^{\text {veto }}=30 \mathrm{GeV}$. By further decreasing $p_{T}^{\text {veto }}$, perturbative contributions beyond the NNLO can become sizeable, since they are enhanced by powers of logarithmic terms of the type $\ln p_{T}^{\text {veto }}$.

In this paper we have limited our analysis to the SM Higgs boson. The NLO QCD corrections to Higgs boson production within the Minimal Supersymmetric extension of the Standard Model (MSSM) are known [14, 41, 9]; for small values $(\tan \beta \lesssim 5)$ of the MSSM parameter $\tan \beta$, they are comparable to those for the SM Higgs. This suggests that this similarity of the size of the perturbative QCD corrections remains true also at NNLO.

In actual experiments at hadron colliders, the observed cross section for Higgs signal events can significantly depend on the details of the experimental analysis. This is particularly true when a jet veto procedure is applied, because of effects due to efficiency for jet reconstruction, jet energy calibration, presence of pile-up events and so forth. Quantitative estimates of these effects require event simulations [6, [7] based on parton shower Monte Carlo generators [42, 43]. The parton shower produces multi-parton configurations that approximate the exact QCD matrix elements, but such approximation does not strictly correspond to the perturbative expansion of the cross section at LO, NLO, NNLO and so forth. Therefore, the NLO or NNLO $K$-factors computed in this paper cannot naively be used to rescale the results of present Monte Carlo simulations at the the Tevatron and the LHC. As is customary practice in QCD analyses, more refined studies, which combine the perturbative QCD predictions with the Monte Carlo simulations at the detector level, are necessary to firmly quantify the expected number of veto selected Higgs events at Tevatron and the LHC.

Acknowledgements. Part of this work was performed during the Workshop on 'Physics at TeV Colliders' (Les Houches, France, May 2001). We would like to thank Elzbieta Richter-Was for many useful discussions and comments. We also thank Thomas Gehrmann and Stefano Moretti for comments on the draft. 


\section{References}

[1] For a review on Higgs physics in and beyond the Standard Model, see J. F. Gunion, H. E. Haber, G. L. Kane and S. Dawson, The Higgs Hunter's Guide (Addison-Wesley, Reading, Mass., 1990).

[2] The LEP Collaborations and the LEP Working Group for Higgs boson searches, hepex/0107029.

[3] P. Igo-Kemenes, presentation given at the open session of the LEP Experiments Committee Meeting, 3 November 2000 (see: http://lepHiggs.web.cern.ch/LEPHIGGS/talks/index.htm]).

[4] R. Barate et al., ALEPH Coll., Phys. Lett. B 495 (2000) 1; M. Acciarri et al., L3 Coll., Phys. Lett. B 495 (2000) 18; P. Abreu et al., DELPHI Coll., Phys. Lett. B 499 (2001) 23; G. Abbiendi et al., OPAL Coll., Phys. Lett. B 499 (2001) 38; P. Achard et al., L3 Coll., Phys. Lett. B 517 (2001) 319.

[5] The LEP Collaborations, the LEP Electroweak Working Group and the SLD Heavy Flavour and Electroweak Working Group, CERN report LEPEWWG/2001-01.

[6] M. Carena et al., Report of the Tevatron Higgs Working Group, hep-ph/0010338.

[7] CMS Coll., Technical Proposal, report CERN/LHCC/94-38 (1994); ATLAS Coll., ATLAS Detector and Physics Performance: Technical Design Report, Volume 2, report CERN/LHCC/99-15 (1999).

[8] H. M. Georgi, S. L. Glashow, M. E. Machacek and D. V. Nanopoulos, Phys. Rev. Lett. 40 (1978) 692.

[9] M. Spira, Fortsch. Phys. 46 (1998) 203.

[10] C. A. Nelson, Phys. Rev. D 37 (1988) 1220; M. Dittmar and H. Dreiner, Phys. Rev. D 55 (1997) 167.

[11] T. Han and R. Zhang, Phys. Rev. Lett. 82 (1999) 25; T. Han, A. S. Turcot and R. Zhang, Phys. Rev. D 59 (1999) 093001.

[12] S. Dawson, Nucl. Phys. B 359 (1991) 283.

[13] A. Djouadi, M. Spira and P. M. Zerwas, Phys. Lett. B264 (1991) 440.

[14] M. Spira, A. Djouadi, D. Graudenz and P. M. Zerwas, Nucl. Phys. B453 (1995) 17.

[15] M. Kramer, E. Laenen and M. Spira, Nucl. Phys. B 511 (1998) 523.

[16] S. Catani, D. de Florian and M. Grazzini, JHEP 0105 (2001) 025.

[17] R. V. Harlander and W. B. Kilgore, Phys. Rev. D 64 (2001) 013015.

[18] R. V. Harlander, Phys. Lett. B492 (2000) 74.

[19] J. M. Campbell and E. W. Glover, Nucl. Phys. B 527 (1998) 264. 
[20] S. Catani and M. Grazzini, Nucl. Phys. B570 (2000) 287.

[21] Z. Bern, V. Del Duca and C. R. Schmidt, Phys. Lett. B445 (1998) 168; Z. Bern, V. Del Duca, W. B. Kilgore and C. R. Schmidt, Phys. Rev. D 60 (1999) 116001.

[22] S. Catani and M. Grazzini, Nucl. Phys. B591 (2000) 435.

[23] T. Matsuura, S. C. van der Marck and W. L. van Neerven, Nucl. Phys. B319 (1989) 570.

[24] A. D. Martin, R. G. Roberts, W. J. Stirling and R. S. Thorne, Eur. Phys. J. C18 (2000) 117.

[25] W. L. van Neerven and A. Vogt, Phys. Lett. B490 (2000) 111, Nucl. Phys. B588 (2000) 345.

[26] S. Catani, D. de Florian and M. Grazzini, preprint CERN-TH/2001-147 hep-ph/0106049, presented at the 36th Rencontres de Moriond on QCD and Hadronic Interactions, Les Arcs, France, 17-24 March 2001 and at the 9th International Workshop on Deep Inelastic Scattering (DIS 2001), Bologna, Italy, 27 April - 1 May 2001.

[27] D. de Florian, M. Grazzini and Z. Kunszt, Phys. Rev. Lett. 82 (1999) 5209.

[28] J. Ellis, M.K. Gaillard and D.V. Nanopoulos, Nucl. Phys. B106 (1976) 292; A. Vainshtein, M. Voloshin, V. Zakharov and M. Shifman, Sov. J. Nucl. Phys. 30 (1979) 711 [Yad. Fiz. 30 (1979) 1368].

[29] K. G. Chetyrkin, B. A. Kniehl and M. Steinhauser, Phys. Rev. Lett. 79 (1997) 353, Nucl. Phys. B 510 (1998) 61.

[30] A. P. Contogouris, N. Mebarki and S. Papadopoulos, Int. J. Mod. Phys. A 5 (1990) 1951.

[31] M. Gluck, E. Reya and A. Vogt, Eur. Phys. J. C5 (1998) 461.

[32] H. L. Lai et al. [CTEQ Coll.], Eur. Phys. J. C 12, 375 (2000).

[33] J. Huston, S. Kuhlmann, H. L. Lai, F. Olness, J. F. Owens, D. E. Soper and W. K. Tung, Phys. Rev. D 58 (1998) 114034.

[34] R. V. Harlander and W. B. Kilgore, preprint BNL-HET-01-36 hep-ph/0110200.

[35] C. R. Schmidt, Phys. Lett. B 413 (1997) 391.

[36] S. Dawson and R. P. Kauffman, Phys. Rev. Lett. 68 (1992) 2273. R. P. Kauffman, S. V. Desai and D. Risal, Phys. Rev. D 55 (1997) 4005 [Erratum ibid. D 58 (1997) 119901].

[37] R. K. Ellis, I. Hinchliffe, M. Soldate and J. J. van der Bij, Nucl. Phys. B 297 (1988) 221; U. Baur and E. W. Glover, Nucl. Phys. B 339 (1990) 38.

[38] V. Del Duca, W. Kilgore, C. Oleari, C. Schmidt and D. Zeppenfeld, preprint MADPH-01-1226 hep-ph/0105129, preprint MADPH-01-1235 hep-ph/0108030.

[39] S. Frixione, Z. Kunszt and A. Signer, Nucl. Phys. B 467 (1996) 399.

[40] S. Catani, hep-ph/9709503, in Proc. of the 32nd Rencontres de Moriond: QCD and HighEnergy Hadronic Interactions, ed. J. Tran Than Van (Editions Frontières, Gif-sur-Yvette, 1997) p. 331. 
[41] S. Dawson, A. Djouadi and M. Spira, Phys. Rev. Lett. 77 (1996) 16.

[42] G. Marchesini, B. R. Webber, G. Abbiendi, I. G. Knowles, M. H. Seymour and L. Stanco, Comput. Phys. Commun. 67 (1992) 465; G. Corcella, I.G. Knowles, G. Marchesini, S. Moretti, K. Odagiri, P. Richardson, M.H. Seymour and B.R. Webber, JHEP 0101 (2001) 010.

[43] T. Sjostrand, P. Eden, C. Friberg, L. Lonnblad, G. Miu, S. Mrenna and E. Norrbin, Comput. Phys. Commun. 135 (2001) 238. 